Article

\title{
In Situ Polymerization of Chiral Poly(fluorene-alt-benzothiadiazole) Nanocomposites with Enhanced Chirality
}

\author{
Hongsub Jee ${ }^{1}$, Hyeong-Sub $\mathrm{Oh}^{2}$ and Jaehyeong Lee ${ }^{3, *(1)}$ \\ 1 College of Information and Communication Engineering, Sungkyunkwan University, Suwon 16419, Korea; \\ hsjee@skku.edu \\ 2 Get Smart Materials, Daejeon 34054, Korea; hsoh5191@gmail.com \\ 3 Department of Electrical and Computer Engineering, Sungkyunkwan University, Suwon 16419, Korea \\ * Correspondence: jaehyeong@skku.edu; Tel.: +82-31-299-4950
}

Received: 26 October 2020; Accepted: 4 December 2020; Published: 6 December 2020

\begin{abstract}
Poly(fluorene-alt-benzothiadiazole) (PFBT) is a promising chiral polymer for use in metamaterials and other photonic applications, due to its large chiral optical activity at visible wavelengths. However, its usages are very limited, since it is not readily patternable into microstructures and challenging to apply to applications. In this paper, we demonstrate photo patterning of chiral PFBT/Achiral SU-8 photoresist to produce high quality structures while retaining the extraordinary chiral optical activity of our previously-reported PFBT/SU8 nanocomposite films. The ability to produce cleanly patterned microfeatures with high chirality may enable wider use of PFBT in chiral metamaterials and other photonic applications.
\end{abstract}

Keywords: polymerization; fabrication; chirality; nanocomposites

\section{Introduction}

The research field of chiral photonics is an area of growing interest, with potential to contribute to applications ranging from negative- and near-zero refractive index metamaterials [1,2], to chiral bioimaging [3,4], to all-optical switching [5-8]. Realization of such applications, and the full potential of this field, demands materials with dramatically greater optical chirality than that observed in naturally occurring materials [9-11]. A key strategy for increasing chirality is coupling across length scales, from molecular chirality to the geometric chirality of nano- and microscale patterned features [12]. Here, we present a material and process that allows high fidelity photopatterning of a polymer nanocomposite (chiral poly(fluorene benzothiadiazole in SU-8 photoresist)) with extraordinarily high chiro-optical activity. Circular dichroism exceeding one degree, at visible wavelengths, is produced by a thin film of this nanocomposite. Conventional photolithography can be used to produce patterns in this material with sharp edges and no residual material between patterned features. This adds a valuable new tool to the palette of materials and processes available for pursuing chiral photonics.

Although biological materials are predominantly chiral, the field of chiral photonics requires new, synthetic materials with much higher chiro-optical activity than natural materials. In particular, metamaterials with negative or near-zero refractive index have attracted much attention in recent years, because of their potentially revolutionary impact in applications including sub-wavelength imaging [13], data storage [14], optical switching [15], cloaking [16,17], and stealth technology [18,19]. Typically, in negative index materials (NIMs), the electric permittivity, $\varepsilon$, and the magnetic permeability, $\mu$, are both made negative by using an array of sub-wavelength oscillators to achieve negative permeability [20]. Fabrication of such "double negative" NIMs poses extreme technological challenges. 
An alternative approach to achieving a negative refractive index employs chiral materials with high optical activity [21]. In a chiral material, the effective refractive index is given by

$$
n_{e f f}=\sqrt{\varepsilon \mu} \pm \kappa
$$

where $\mathrm{K}$ is the chirality parameter, and the effective refractive index is increased for light of one circular polarization and decreased for light of the other circular polarization [22]. As shown by Equation (1), for a sufficiently large value of $k$ and a sufficiently small value of $\varepsilon$, the effective refractive index for one circular polarization could become negative even if $\mu$ remained near one. Although most biological materials are chiral, the chirality parameter in naturally-occurring materials is typically below 0.001 [23], far too small to make substantive contributions to the effective refractive index. Moreover, most naturally-occurring materials have a maximum chiro-optical activity at ultraviolet wavelengths. In contrast, co-polymers with a donor-acceptor structure such as chiral poly(fluorene-alt-benzothiadiazole) (PFBT), can have optical transitions in the visible spectral regime and exhibit maximum values of $k$ at visible wavelengths [24]. Along with their potential for use in this chiral route to negative-index metamaterials, such highly optically active materials also show promise for other interesting chiral photonic devices such as polarization-sensitive phase-shifters.

We have previously demonstrated the plasmonic enhancement of chirality in PFBT films using gold nanoparticles [25], and more recently, we reported on thin film fabrication of nanocomposites of a PFBT in a photopolymer matrix [26]. This optically clear nanocomposite shows exceptionally large circular dichroism produced by the enhanced helical conformation of PFBT within the fully cured SU-8 matrix. These chiral PFBT/SU-8 nanocomposites exhibit exceptionally high circular dichroism (CD) (dissymmetry ratio of gabs $=-0.32$ ), two orders of magnitude larger than that of pure PFBT films of the same optical density (gabs $=-0.003$ ). SU-8 is well known for its processibility and its outstanding chemical and mechanical properties (chemical resistance, mechanical strength, high temperature strength, thermal stability, and biocompatibility), which have made it the material of choice for MEMS and related applications. As described above, the dramatic enhancement of $C D$ was achieved in PFBT/SU-8 nanocomposites after ultraviolet (UV) light exposure, development, and annealing. However, using a typical SU-8 processing and development process, the PFBT/SU-8 nanocomposite film was not fully removed from the nominally unexposed area. A thin film of residue remained in the regions between patterned structures. Aggregates of PFBT were clearly visible within the SU-8 matrix in this residual film. Although topographic patterning was achieved in that work, with a thick film in exposed areas and much thinner film in unexposed areas, both the exposed and unexposed areas contributed to the overall chiro-optical response. The chirality contrast between exposed and unexposed regions was necessarily smaller than it would be for a cleanly patterned film with bare substrate between the patterned features. In this paper, we demonstrate the fabrication of cleanly-patterned PFBT/SU-8 nanocomposite structures that still exhibit dramatically enhanced optical activity compared with pure PFBT film, even in the absence of a residual film of chiral nano-aggregates between the patterned structures.

\section{Materials and Methods}

Raw Materials: SU-8 2025 resist was purchased from Microchem. Propylene glycol methyl ether acetate (PGMEA), tetrahydrofuran (THF), and isopropanol alcohol (IPA) were purchased from Sigma-Aldrich. All chemicals were used as received.

Synthesis of PFBT and preparation of PFBT/SU-8 mixture: We introduced chirality in PFBT by attaching (S)-3,7-dimethyloctyl substituents at the 9-position of the fluorene monomer. Chiral PFBT was synthesized using palladium-catalyzed Suzuki polycondensation and the product was purified with Soxhlet extraction to obtain high molecular weight $(\mathrm{Mn}=13,000, \mathrm{Mw}=30,000)$. The PFBT was dissolved in THF by stirring with a magnetic stirrer for $\sim 24 \mathrm{~h}$. SU-8 2025 was then added to the PFBT/THF solution and stirred for an additional $24 \mathrm{~h}$ with a magnetic stirrer. 
Fabrication: Figure 1 shows the overall process for photopatterning of the PFBT/SU-8 2025 nanocomposite. The PFBT/SU-8 2025 solution, prepared as described above, was spin-coated at $1000 \mathrm{RPM}$ onto a glass substrate, then baked at $65^{\circ} \mathrm{C}$ for $60 \mathrm{~min}$, then baked at $95^{\circ} \mathrm{C}$ for $10 \mathrm{~min}$, and finally allowed to cool to room temperature for $2 \mathrm{~h}$. A Karl Suss UV aligner ( $365 \mathrm{~nm}$ wavelength) and chrome photo mask were used for the UV exposure process with a total exposure dose of $225 \mathrm{~mJ} / \mathrm{cm}^{2}$. After exposure, samples were baked at $65{ }^{\circ} \mathrm{C}$ for $10 \mathrm{~min}$, then at $95{ }^{\circ} \mathrm{C}$ for $10 \mathrm{~min}$ to accelerate the crosslinking of the polymer. They were allowed to cool for $2 \mathrm{~h}$ to room temperature before development. To develop the UV exposed samples, they were submerged in PGMEA for $5 \mathrm{~h}$ to remove unpolymerized material. IPA and deionized water were used to rinse the developed samples. After development and rinsing, samples were annealed at $150^{\circ} \mathrm{C}$ for $15 \mathrm{~min}$ in an inert atmosphere glove box, then gradually cooled to room temperature.

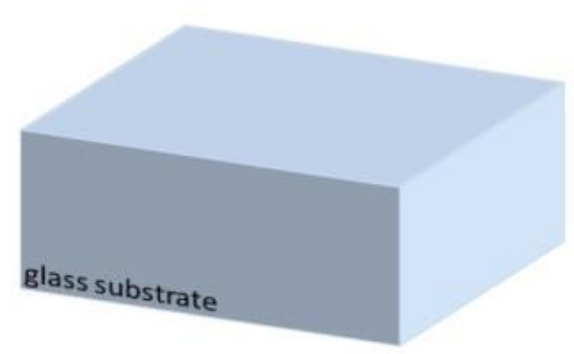

(a) cleaning glass substrate

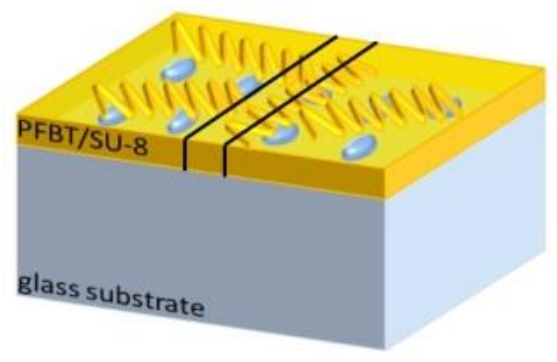

(c) exposure and post baking

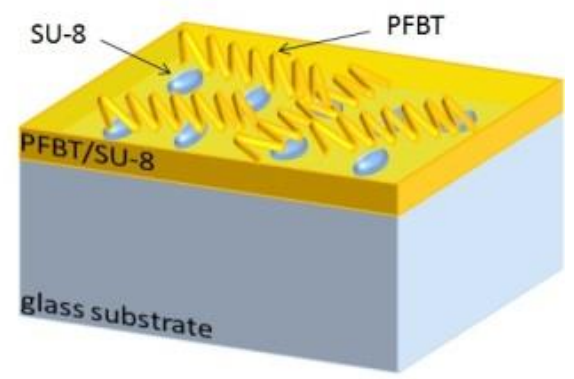

(b) PFBT/SU-8 coating and soft baking

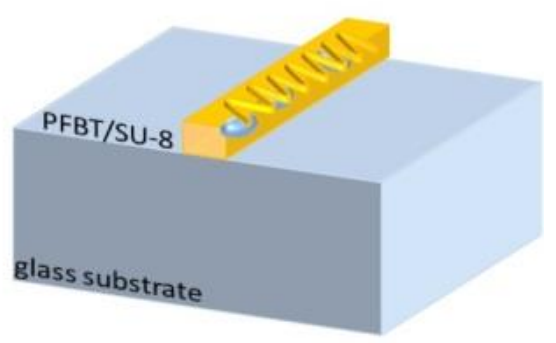

(d) development and rinse

Figure 1. Photofabrication process for poly(fluorene-alt-benzothiadiazole)(PFBT/SU-8) nanocompositemicrostructures.

Characterization. The patterned structures were analyzed by scanning electron microscopy (SU-4000, Hitachi, Japan) and circular dichroism (CD) spectroscopy (J-815, Jasco, UK).

\section{Results and Discussions}

Figure 2A shows patterned PFBT/SU-8 structures after the UV exposure and development process presented in our previous work [26]. As shown schematically in Figure 2B, the material in nominally unexposed (nominally unpolymerized) areas was not completely removed, and a residual film remained between the patterned features. This suggests that PFBT, a strong absorber, served to increase the photosensitivity of the film. Use of a high exposure dose (overexposure) and leakage of light under the photomask, due to imperfect contact of the photomask with the film, may cause unwanted crosslinking of the polymer. Heating, due to absorption by PFBT, could also initiate unwanted cross-linking. That unintentionally-cross-linked material was then not removed during development. To achieve a clean photo patterning, without any residual film between patterned features, we ensured the good contact of the photomask, optimized the photoresist composition, and optimized the UV exposure dose. Because of the different molecular sizes and coefficients of thermal expansion of PFBT and SU-8, making PFBT/SU-8 films with smooth surfaces was challenging. Smooth films were produced by first baking the PFBT/SU- 8 film at $65{ }^{\circ} \mathrm{C}$ for $60 \mathrm{~min}$ then at $95^{\circ} \mathrm{C}$ for $10 \mathrm{~min}$. This pre-baking step 
reduces the solvent concentration in the spin-coated PFBT/SU-8 layer and $60 \mathrm{~min}$ of pre-baking time in a semifluid state may promote mixing between PFBT and SU-8. With smoother, flatter films from this improved pre-bake process, better contact between the photomask and substrate was achieved during UV exposure. The ratio of SU-8 to PFBT was also optimized to achieve the highest CD signal while maintaining clean patterning. First, we tested PFBT/SU-8 mass ratios from 1:20 to 1:80 at a fixed solid content of 30\% SU-8. The 1:10 and 1:20 ratios did not form well-mixed films and produced poor quality patterns. Films using a 1:40 ratio could be patterned well and showed higher CD than those using 1:60 and 1:80 ratios. We then fixed the PFBT/SU-8 mass ratio at 1:40 and varied the amount of SU-8 (solid content) in the solution used for spin-coating. For solid content of $20 \%$ or less, well-defined patterns could not be obtained. Films prepared using $40 \%$ solid content SU-8 showed lower CD than those spun from 30\% solid content. Thus, a 1:40 PFBT:SU8 mass ratio and 30\% SU-8 were selected as the best combination for good quality patterning and high CD. The UV exposure dose of $225 \mathrm{~mJ} / \mathrm{cm}^{2}$ used here is about half the dose used in our previous work. Figure 2D shows an SEM image of a single feature from a photo patterned chiral polymer nanocomposite produced using these optimized conditions. Note that the unexposed area, inside the ring, was perfectly removed and the boundary of the structure was cleanly fabricated as well, in contrast to the structure shown in Figure 2C, produced using the same mask.
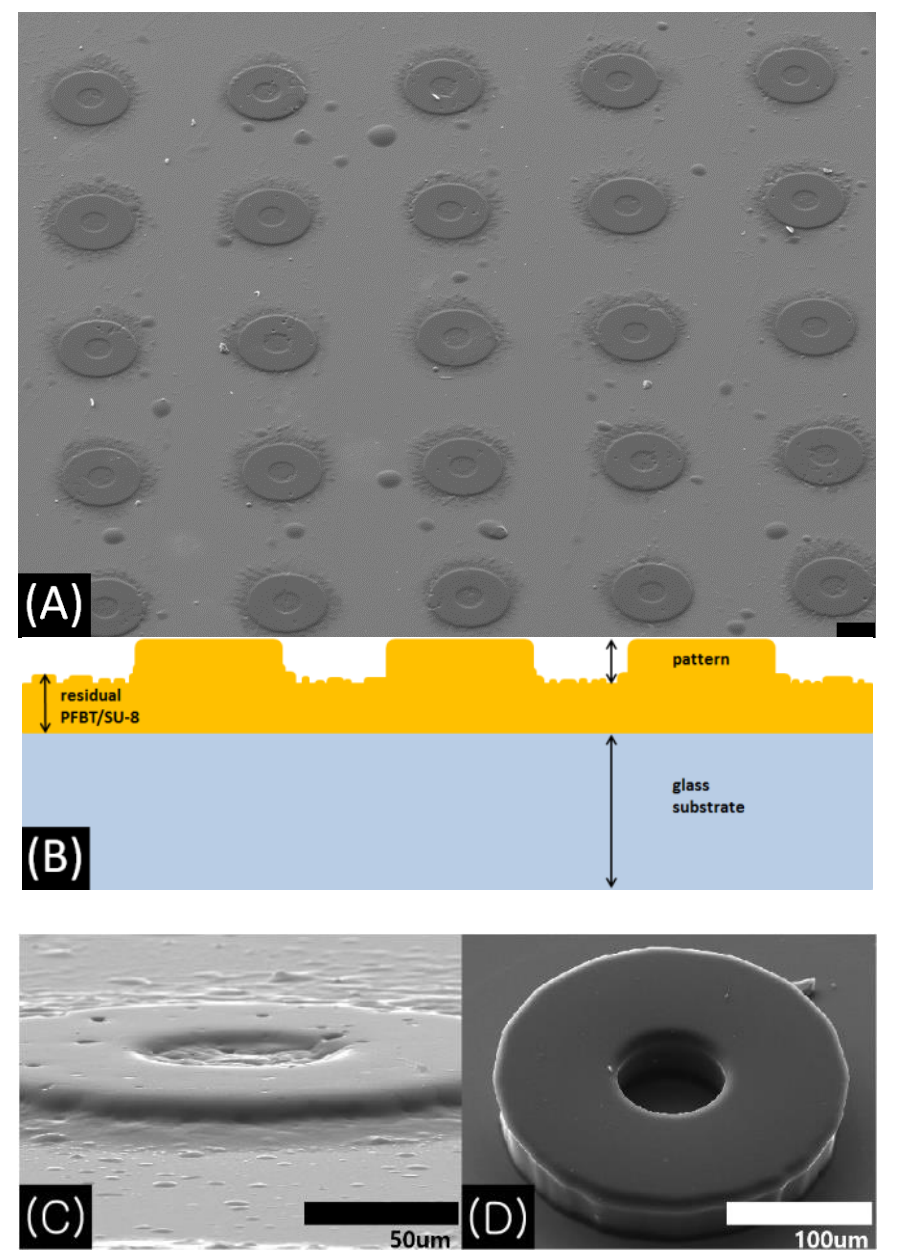

Figure 2. PFBT/SU-8 pattern using the standard SU-8 exposure and development process as reported in our previous work [26]. Black bar scale $=100 \mu \mathrm{m}$. (A) Top view scanning electron microscope (SEM) image, (B) schematic cross sectional view of patterned PFBT/SU-8. (C) Higher magnification, low angle SEM picture of the sample shown in (A), and (D) higher magnification SEM image of a sample produced by the optimized method presented here, patterned with the same mask. 
Figure 3 shows various patterns produced in this PFBT/SU-8 nanocomposite at the same optimized conditions. In all cases, the patterns were clean, high-fidelity replicas of the mask used to create them. The patterned structures are flat and uniform, showing no evidence of PFBT aggregation, and suggesting that the PFBT and SU-8 are well mixed at a molecular level in these patterned films.
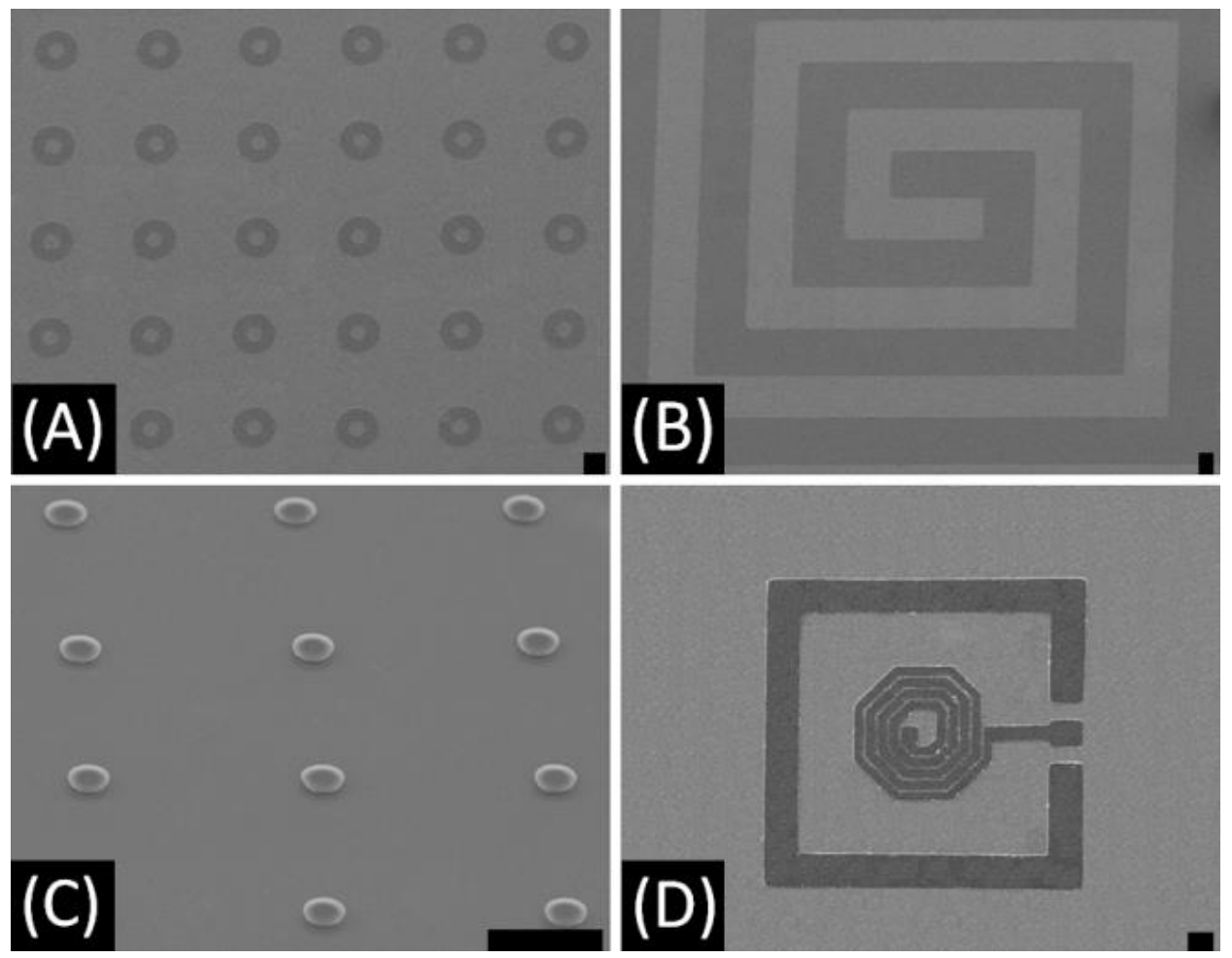

Figure 3. SEM images of patterned chiral polymer. Black bar scale $=100 \mu \mathrm{m}$. (A) Ring shape, (B) spiral shape, (C) spheroid shape, (D) inductor shape.

To investigate the $\mathrm{CD}$ enhancement, we compared the effect of annealing on the UV-visible absorption and CD spectra of pure PFBT and PFBT/SU-8 photopatterned films. The UV-visible absorption spectra of the PFBT/SU-8 samples were similar to that of the pure PFBT film. However, the patterned PFBT/SU-8 showed much larger CD than the pure PFBT film (Figure 4). After annealing, the maximum CD signal from the patterned film was 30 times that of the pure chiral PFBT film of the same optical absorbance (Figure 4B). We attribute this to the local changes in the PFBT configuration. However, the CD of the cleanly patterned PFBT/SU-8 was lower than that of a PFBT/SU-8 sample patterned as described in our previous work (Figure 4B). The peak value of CD ( $-789 \mathrm{mdeg}$ ) was $42 \%$ of the corresponding peak value for the pattern with residual film $(-1890 \mathrm{mdeg})$.

In the measurement of the $\mathrm{CD}$ spectrum, linear dichroism and linear birefringence frequently make a huge artifact especially in the polymer film or crystal sample [27]. To verify the measured CD spectrum, we remeasured the PFBT/SU-8 cleanly patterned film and annealed PFBT/SU-8 cleanly patterned film by CD spectrometer with different orientations. As shown in Figure 5, remeasured values were very similar compared to the original values. 

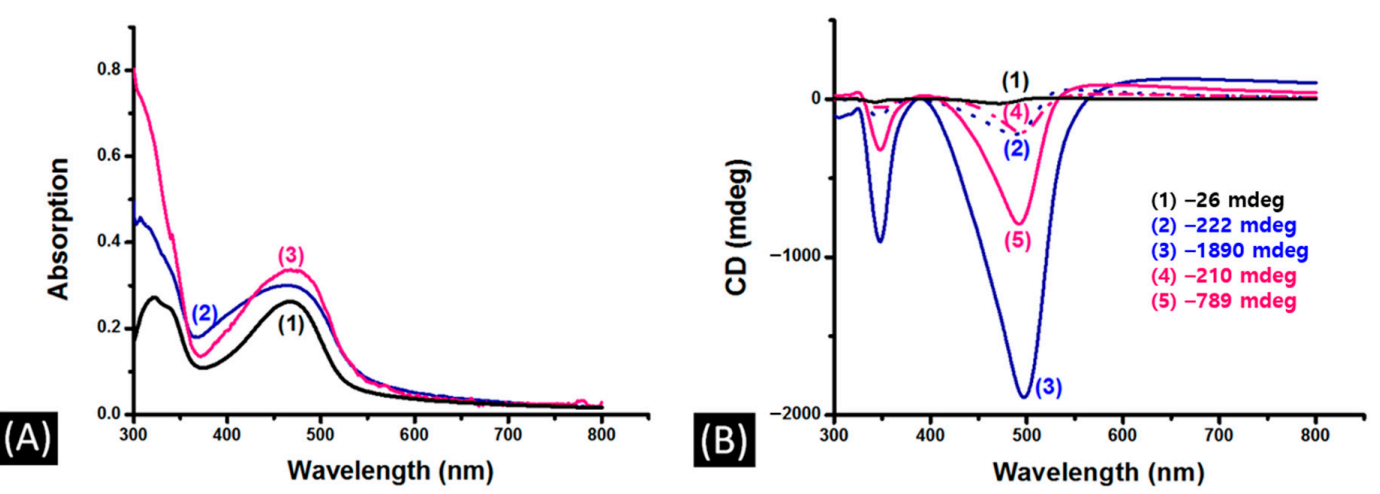

Figure 4. (A) UV absorption and (B) circular dichroism (CD) of chiral polymer films: (A-1) annealed pure PFBT film, (A-2) annealed PFBT/SU-8 patterned film with residual PFBT/SU-8, processed as in our previous work [26], (A-3) annealed PFBT/SU-8 film patterned cleanly, using optimized conditions, (B) (B-1) annealed pure PFBT film, (B-2) PFBT/SU-8 patterned film with residual PFBT/SU-8, (B-3) annealed PFBT/SU-8 patterned film with residual PFBT/SU-8, (B-4) PFBT/SU-8 cleanly patterned film, (B-5) annealed PFBT/SU-8 cleanly patterned film. All patterned samples were 1:40 (PFBT:SU-8) mass ratio films spin-coated from $30 \%$ solid content SU-8 solution.

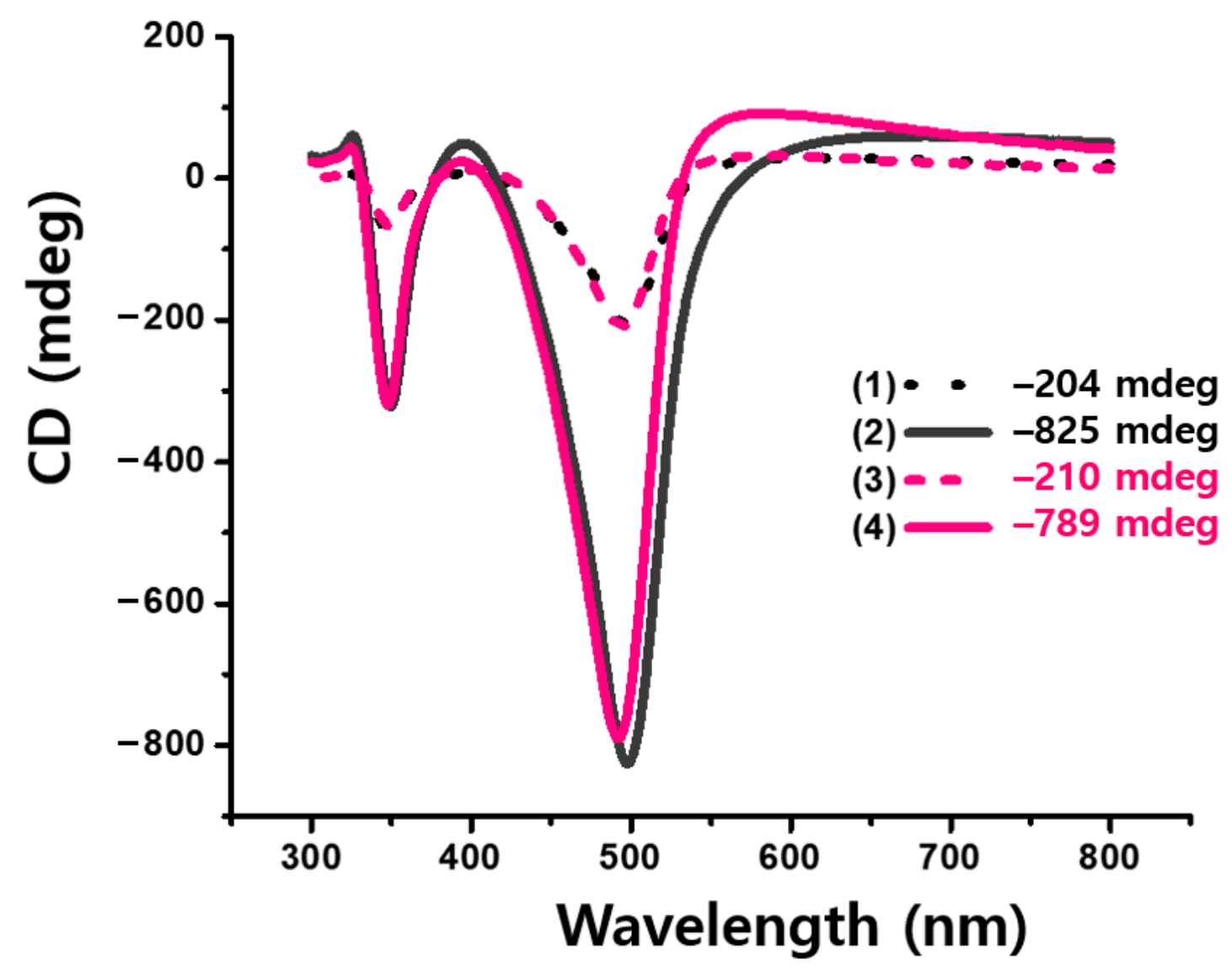

Figure 5. CD of chiral polymer films: (1) remeasured PFBT/SU-8 cleanly patterned film, (2) remeasured annealed PFBT/SU-8 cleanly patterned film, (3) PFBT/SU-8 cleanly patterned film, (4) annealed PFBT/SU-8 cleanly patterned film.

While graph (4) shows -789 mdeg, the remeasured value shows -825 mdeg, which is slightly increased as shown, in graph (2), and the results show that the measured CD spectrum of PFBT/SU-8 cleanly patterned films were obtained accurately. All these measured spectra show that the residual film in the nominally unpolymerized regions makes a substantial contribution to the 
CD. We suggest that the aggregation state of PFBT can change more freely during annealing in the thinner, residual film, leading to increased helical organization. As a result, the areas with residual film could have even higher chirality than the thicker areas. Additional ordering could be introduced by the interfaces. Nonetheless, the cleanly patterned film still shows extraordinarily high CD. Moreover, the cleanly patterned films have a clear difference in chirality between the exposed and unexposed areas. This difference in chiro-optic properties between regions may be more important than the absolute value of the chirality parameter in applications.

\section{Conclusions}

In summary, we demonstrated the fabrication of chiral PFBT/achiral SU-8 microstructures with a good contact of photomask, optimum photoresist composition, and optimum exposure dosage, enabling high-fidelity patterning of sharp features in this polymer nanocomposite. Patterned PFBT/SU-8 structures showed extraordinarily high circular dichroism, 30 times that of a pure PFBT film with same UV absorbance. This enhancement is due to the local changes in PFBT configuration with UV exposure and annealing. Because PFBT is a strong absorber that changes the photosensitivity of the film and because its long polymer chains can change the morphology of the film, optimizing the composition of the PFBT/SU8 chiral photoresist formulation is essential. Moreover, this clean patterning allows us to separate out the contribution of the residual film present between features in our prior work. The ability of cleanly photopatterned chiral materials can enable the coupling of chirality across multiple length scales because it allows planar chiral elements to be written from intrinsically chiral materials.

Author Contributions: Conceiving the idea, H.J.; designing the study, H.J., H.S.O.; experiments, H.J., H.S.O.; data analysis, H.J., H.S.O.; writing-original draft, H.J., writing-review and editing, H.J., J.L.; supervision, J.L. All authors have read and agreed to the published version of the manuscript.

Funding: This work was partially supported by the National Research Foundation of Korea (NRF) grant funded by the Korea government (MSIT) (No. NRF-2019R1F1A1061615) and Korea Electric Power Corporation. (Grant number: R17XA05-1).

Conflicts of Interest: The authors declare no conflict of interest.

\section{References}

1. Foteinopoulou, S.; Economou, E.N.; Soukoulis, C.M. Refraction in media with a negative refractive index. Phys. Rev. Lett. 2003, 90, 107402. [CrossRef]

2. Rhelby, R.A.; Smith, D.R.; Schultz, S. Experimental veripcation of a negative index of refraction. Science 2001, 292, 77-79. [CrossRef]

3. Koutsoupidou, M.; Uzunoglu, N.; Karanasiou, I.S. Antennas on metamaterial substrates as emitting components for THz biomedical imaging. In Proceedings of the 2012 IEEE 12th International Conference on Bioinformatics \& Bioengineering (BIBE), Larnaca, Cyprus, 11-13 November 2012; Institute of Electrical and Electronics Engineers (IEEE): Piscataway, NJ, USA, 2012; pp. 319-322. [CrossRef]

4. Jin, Y. Engineering plasmonic gold nanostructures and metamaterials for biosensing and nanomedicine. Adv. Mater. 2012, 24, 5153-5165. [CrossRef] [PubMed]

5. Drachev, V.P.; Cai, W.; Chettiar, U.; Yuan, H.-K.; Sarychev, A.K.; Kildishev, A.V.; Klimeck, G.; Shalaev, V.M. Experimental verification of an optical negative-index material. Laser Phys. Lett. 2006, 3, 49-55. [CrossRef]

6. Shalaev, V.M. Optical negative-index metamaterials. Nat. Photonics 2007, 1, 41-48. [CrossRef]

7. Soukoulis, C.M.; Kafesaki, M.; Economou, E.N. Negative-index materials: New frontiers in optics. Adv. Mater. 2006, 18, 1941-1952. [CrossRef]

8. Valentine, J.; Zhang, S.; Zentgraf, T.; Ulin-Avila, E.; Genov, D.A.; Bartal, G.; Zhang, X. Three-dimensional optical metamaterial with a negative refractive index. Nat. Cell Biol. 2008, 455, 376-379. [CrossRef]

9. Zhang, S.; Park, Y.-S.; Li, J.; Lu, X.; Zhang, W.; Zhang, X. Negative refractive index in chiral metamaterials. Phys. Rev. Lett. 2009, 102, 023901. [CrossRef]

10. Plum, E.; Liu, X.-X.; Fedotov, V.A.; Chen, Y.; Tsai, D.P.; Zheludev, N.I. Metamaterials: Optical activity without chirality. Phys. Rev. Lett. 2009, 102, 113902. [CrossRef] 
11. Plum, E.; Zhou, J.; Dong, J.; Fedotov, V.A.; Koschny, T.; Soukoulis, C.M.; Zheludev, N.I. Metamaterial with negative index due to chirality. Phys. Rev. B 2009, 79, 035407. [CrossRef]

12. Furlani, E.P.; Jee, H.S.; Oh, H.S.; Baev, A.; Prasad, P.N. Laser writing of multiscale chiral polymer metamaterials. Adv. Optoelectron. 2012, 2012,1-7. [CrossRef]

13. Pendry, J.B. Negative Refraction Makes a Perfect Lens. Phys. Rev. Lett. 2000, 85, 3966-3969. [CrossRef] [PubMed]

14. Wuttig, M.; Yamada, N. Phase-change materials for rewriteable data storage. Nat. Mater. 2007, 6, 824-832. [CrossRef] [PubMed]

15. Kind, H.; Yan, H.; Messer, B.; Law, M.; Yang, P. Nanowire Ultraviolet Photodetectors and Optical Switches. Adv. Mater. 2002, 14, 158-160. [CrossRef]

16. Cai, W.; Chettiar, U.K.; Kildishev, A.V.; Shalaev, V.M. Optical cloaking with metamaterials. Nat. Photonics 2007, 1, 224-227. [CrossRef]

17. Pendry, J.B. Controlling electromagnetic fields. Science 2006, 312, 1780-1782. [CrossRef] [PubMed]

18. Liu, N.; Guo, H.; Fu, L.; Kaiser, S.; Schweizer, H.; Giessen, H. Three-dimensional photonic metamaterials at optical frequencies. Nat. Mater. 2008, 7,31-37. [CrossRef]

19. Zhu, W.M.; Liu, A.Q.; Zhang, X.M.; Tsai, D.P.; Bourouina, T.; Teng, J.H.; Zhang, X.H.; Guo, H.C.; Tanoto, H.; Mei, T.; et al. Switchable magnetic metamaterials using micromachining processes. Adv. Mater. 2011, 23, 1792-1796. [CrossRef]

20. Veselago, V.G. The electrodynamics of substances with simultaneously negative values of $\varepsilon$ and $\mu$. Soviet Physics Usp. 1968, 10, 509-514. [CrossRef]

21. Litchinitser, N.M.; Gabitov, I.R.; Maimistov, A.I.; Shalaev, V.M. Progress in Optics; Elsevier: Amsterdam, The Netherlands, 2008; pp. 1-67.

22. Grimsdale, A.C.; Leok Chan, K.; Martin, R.E.; Jokisz, P.G.; Holmes, A.B. Synthesis of light-emitting conjugated polymers for applications in electroluminescent devices. Chem. Rev. 2009, 109, 897-1091. [CrossRef]

23. Liu, Y.; Zhang, X. Metamaterials: A new frontier of science and technology. Chem. Soc. Rev. 2011, 40, 2494-2507. [CrossRef] [PubMed]

24. Abbel, R.R.; Schenning, A.P.H.J.; Meijer, E.W. Molecular weight optimum in the mesoscopic order of chiral fluorene (Co)polymer films. Macromolecules 2008, 41, 7497-7504. [CrossRef]

25. Oh, H.S.; Liu, S.; Jee, H.; Baev, A.; Swihart, M.T.; Prasad, P.N. Chiral poly(fluorene-alt-benzothiadiazole) (PFBT) and nanocomposites with gold nanoparticles: Plasmonically and structurally enhanced chirality. J. Am. Chem. Soc. 2010, 132, 17346-17348. [CrossRef] [PubMed]

26. Oh, H.S.; Jee, H.; Swihart, M.T.; Baev, A.; Prasad, P.N. Dramatic structural enhancement of chirality in photopatternable nanocomposites of chiral poly(fluorene-alt-benzothiadiazole) (PFBT) in achiral SU-8 photoresist. Adv. Funct. Mater. 2012, 22, 5074-5080. [CrossRef]

27. Castiglioni, E.; Biscarini, P.; Abbate, S. Experimental aspects of solid state circular dichroism. Chirality 2009, 21, E28-E36. [CrossRef]

Publisher's Note: MDPI stays neutral with regard to jurisdictional claims in published maps and institutional affiliations.

(C) 2020 by the authors. Licensee MDPI, Basel, Switzerland. This article is an open access article distributed under the terms and conditions of the Creative Commons Attribution (CC BY) license (http://creativecommons.org/licenses/by/4.0/). 